\title{
Sensor Array-Architecture and Monitoring
}

\author{
Vishesh $\mathbf{S}^{1}$, Vilas Gupta $\mathbf{S V}^{2}$, Sourav $\mathbf{H A}^{2}$, Saiesh $\mathbf{R}^{3}$, Sahana $\mathrm{KR}^{4}$, Sindhu $\mathbf{P}^{5}$ \\ B.E, Department of Telecommunication Engineering, BNM Institute of Technology, Bangalore, India ${ }^{1}$ \\ Student, Department of Computer Science Engineering, DSCE, Bangalore, India ${ }^{2}$ \\ Student, Department of Telecommunication Communication Engineering, DSCE, Bangalore, India ${ }^{3}$ \\ Student, Department of Electronics and Communication Engineering, BMSCE, Bangalore, India ${ }^{4}$ \\ Student, Department of Medical Electronics Engineering, BMSCE, Bangalore, India ${ }^{5}$
}

\begin{abstract}
A sensor is a device which detects or measures a physical property and records, indicates, or otherwise responds to it. The sensor used in a particular application is sensitive to one or more sources, and when the sensor is exposed to that stimulus/stimuli, this affects the physical, chemical or electromagnetic properties of the sensor which is further processed to a more usable and readable form. Sensor is the heart of a measurement system. It is the first element that comes in contact with environmental variables to sense and generate an output. A sensor can be classified into various categories like active and passive sensors, analog and digital sensors, mechanical sensors, bio sensors, etc. In this paper we are using an array of sensors, and reading data from the sensors simultaneously and displaying it using an android application called "sense graph" which is a scientific third party application developed by our team. We are using an array of similar sensors- Force sensors, which are capable of measuring force applied per unit area. In this paper, we are interested in measuring force/area on the bottom of the human foot and framing conclusions. The measurements are made in $\mathrm{N} / \mathrm{m}^{2}$ at a particular time interval and a graph of $\mathrm{N} / \mathrm{m}^{2} \mathrm{v} / \mathrm{s}$ time (sec) is plotted. There are regions in the foot where pressure could be very high or negligible for a specific posture or movement of the human body.
\end{abstract}

Keywords: measures, indicates, responds, active and passive sensors, third party applications, analog and digital sensors, posture or movement.

\section{INTRODUCTION}

A sensor is a device which detects or is sensitive to particular stimulus or sensitive to more than one stimulus (stimuli) and responds to the same by change in its physical, chemical or electromagnetic properties which is further processed by a signal processing unit into a more usable and readable form. A sensor can be used alone or as an array (in an array). An array of sensors is a series of similar or different sensors connected/monitored simultaneously to accomplish a specific task. A sensor array is deployed in a certain geometric pattern or exposed to the same stimulus to measure different content or element or molecules or chemical/physical properties of that stimulus. In this paper array of force sensors is arranged in a geometric pattern based on the pressure points to be analysed on the surface of the foot bottom of human beings. Each sensors in the array measures the force executed by the foot at a particular point on the surface of the foot bottom (i.e. force per particular area of the foot)

Following are the various criteria to choose a sensor

- Accuracy

- Precision

- Repeatability

- Reproducibility

- Stability

- Error

- Noise

- Drift

- Resolution

- Minimum Detectable Signal

- Calibration curve

- Sensitivity

- Linearity

- Selectivity 
- Hysteresis

- Measurement range

- Response and recovery time

- Environmental compatibility.

Each sensor in the array is given a code or numbered and it represents the measurement at a particular region of the foot. Figure1 shows a sensor array interfaced to the Microcontroller or Microprocessor.

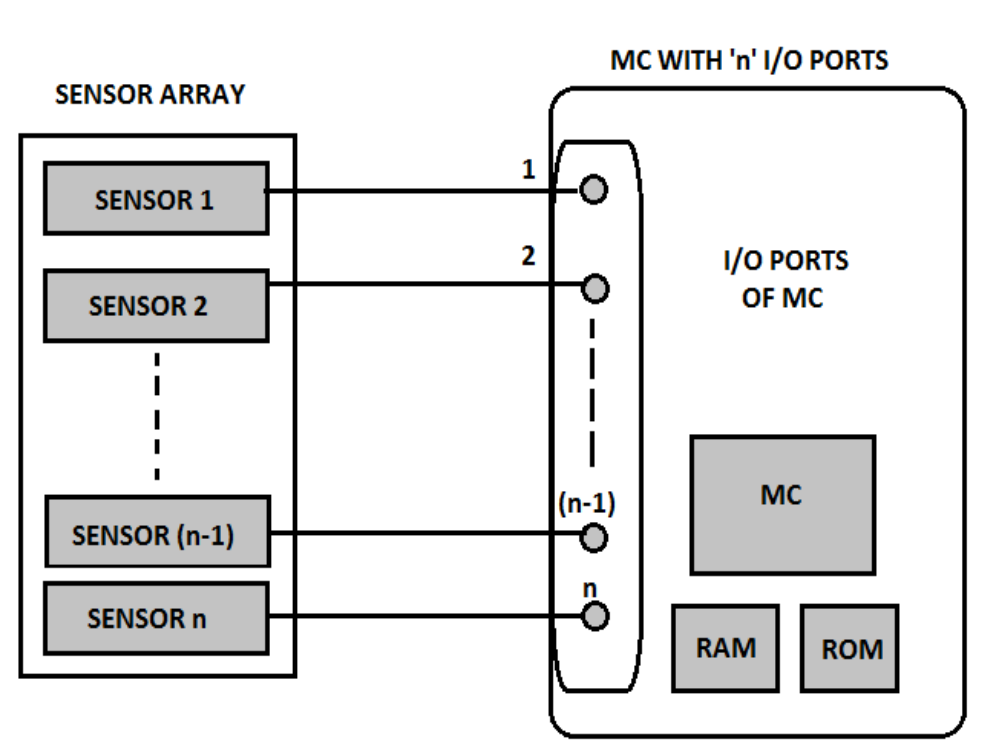

WHERE, $n=1,2,3 \ldots . .$.

SENSOR 1= LOCATED @ REGION 'a1' SENSOR 2= LOCATED @ REGION 'a2'

(I)

SENSOR 1= LOCATED @ REGION 'a(n-1)' SENSOR 1= LOCATED @ REGION 'an'

I/O ports may be analog or digital

Figure1 shows a sensor array interfaced to the Microcontroller or Microprocessor.

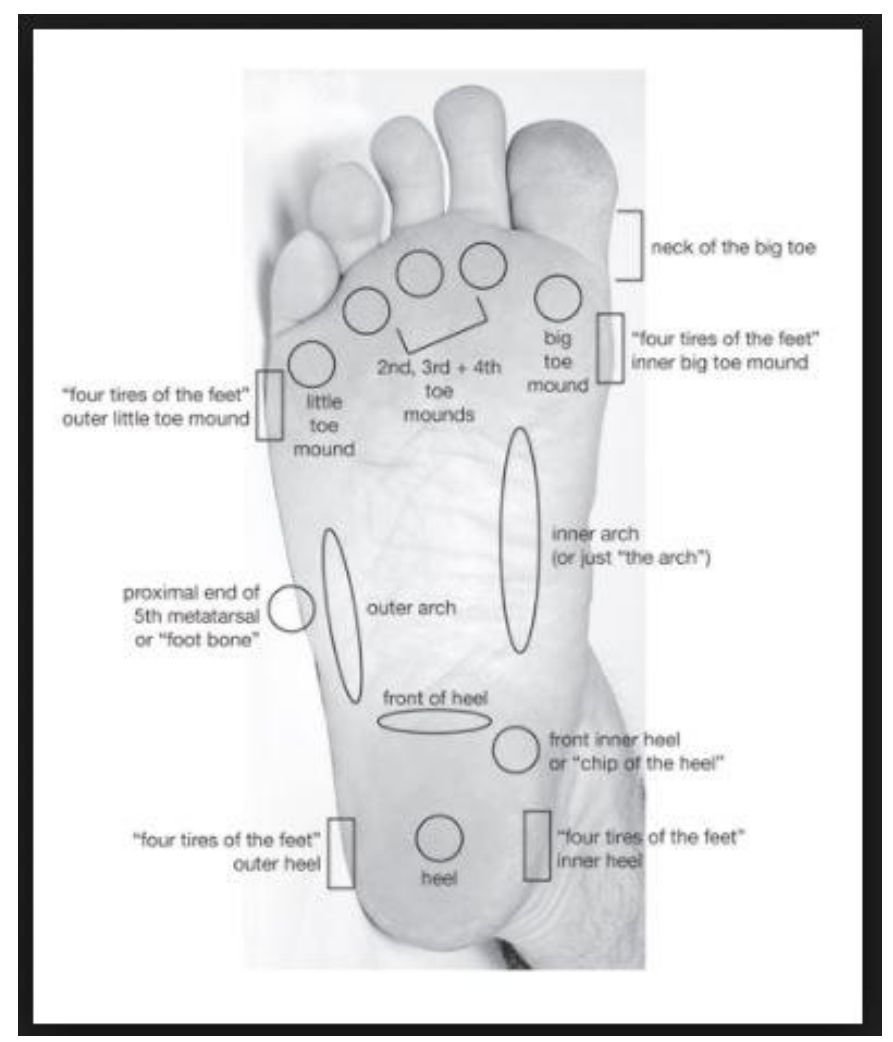

Figure 2 shows the important areas / regions of the human foot bottom. 


\section{STRUCTURE OF BOTTOM FOOT}

A Human foot has a complex design and is unique for each individual. Following are the important areas / regions of the human foot as shown in figure 2

- Toe region (5 toes)

- Neck of these toe regions

- Toe mounds

- Inner arch

- Outer arch

- Proximal end of the fifth metatarsal or "foot bone"

- Front of heel

- Chip of heel

- Heel

- "Four tiers of the feet"-Inner and outer heel.

Each region experiences different Force/unit area or pressure based on the posture- standing, squatting, heels up, toes up etc... and based on the motion of the body.

\section{III.BLOCK DIAGRAM AND METHODOLOGY}

We follow the embedded system life cycle in developing the system, consisting of array of sensors (similar sensors),n I/O pin Microcontroller with a specific RAM and ROM, Bluetooth module for wireless communication and smartphone as the display unit, interpret and recording device. Figure 3 shows the block diagram of our device. In this project we have used three different sized force sensors. Each sensor used has a different area.

The three sensors used are

- Force sensor with circular active area and active sensing area of $0.2 "[5.0 \mathrm{~mm}]$ diameter.

- Force sensors with circular active area and active sensing area of $0.5 "$ " $[12.7 \mathrm{~mm}]$ diameter.

- Square active sensing area of $1.5 "[38.1 \mathrm{~mm}]^{*} 1.5 "[38.1 \mathrm{~mm}]$.

Figure 4 and Figure 5 shows the force sensors with circular active area and square active area respectively.

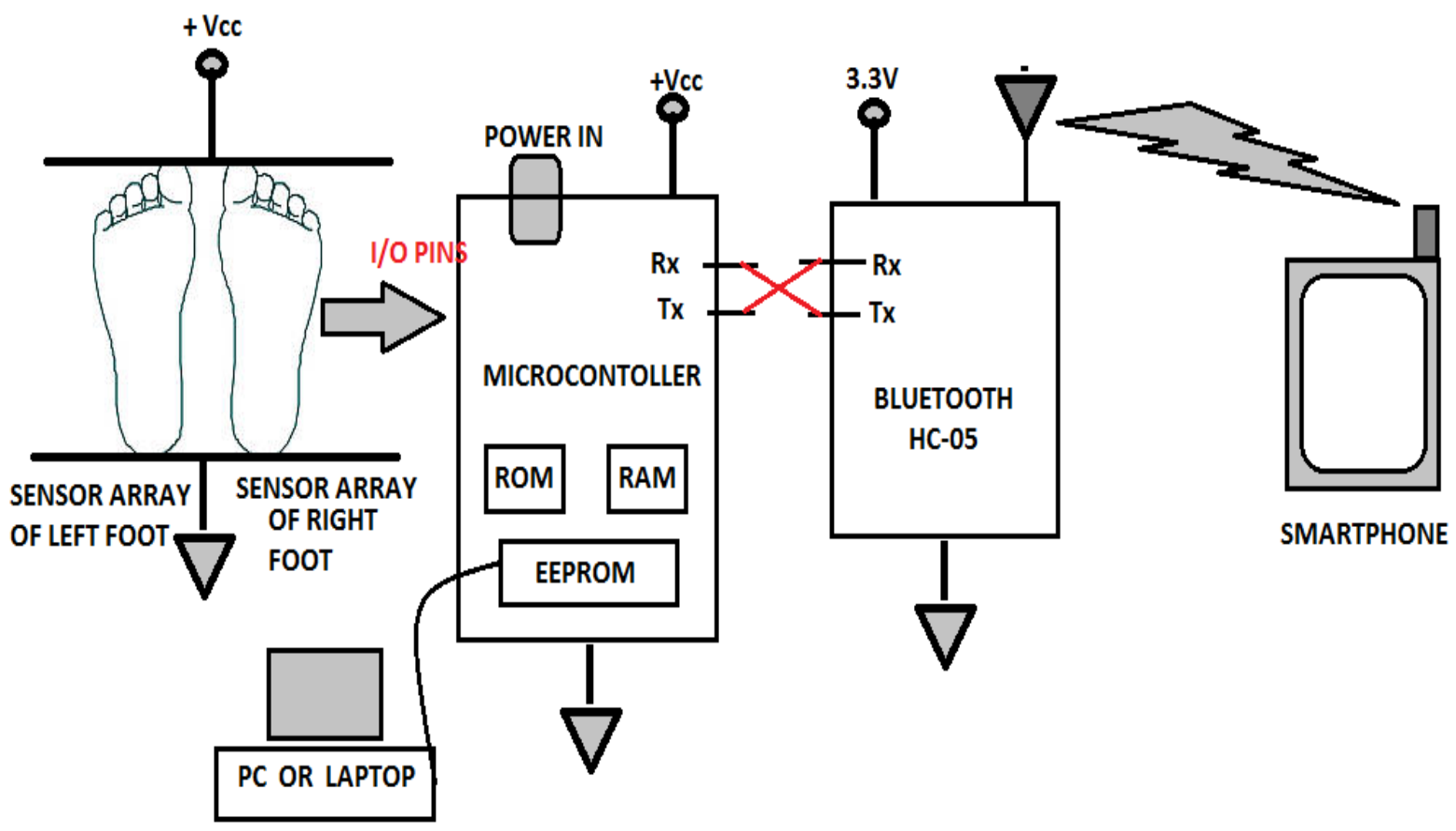

Figure 3 Block diagram 


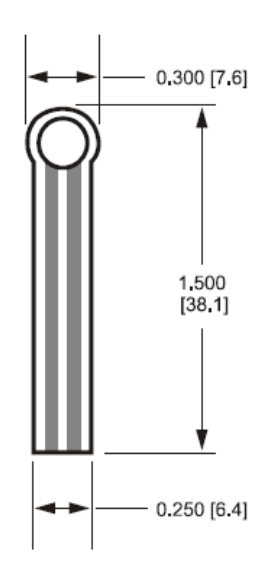

Active Area: $0.2 "[5.0]$ diameter

Nominal Thickness: $0.012 "[0.30 \mathrm{~mm}]$

Material Build:

Semiconductive layer

0.004 " [0.10] PES

Spacer adhesive

$0.002 "$ [0.05] Acrylic

Conductive layer

0.004 " [0.10] PES

Rear adhesive

$0.002 "$ " [0.05] Acrylic

Connector options

a. No connector

b. Solder Tabs (not shown)

c. AMP Female connector

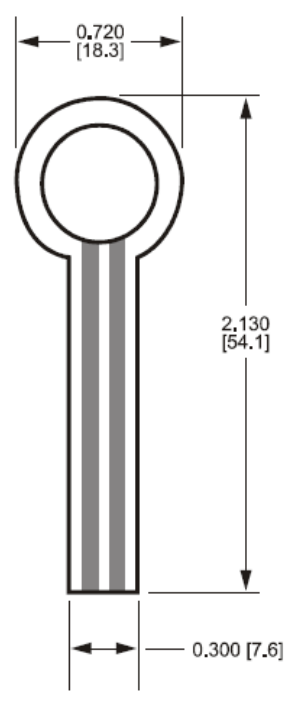

Active Area: $0.5 \%[12.7]$ diameter

Nominal thickness: $0.018^{\circ}[0.46 \mathrm{~mm}]$

Material Build:

Semiconductive Layer

0.005 " [0.13] Ultem

Spacer Adhesive

0.006 " [0.15] Acrylic

Conductive Layer

0.005 " [0.13] Ultem

Rear Adhesive

$0.002 "$ " [0.05] Acrylic

Connector

a. No connector

b. Solder Tabs (not shown)

c. AMP Female connector

Figure 4 shows the force sensors with circular active area.

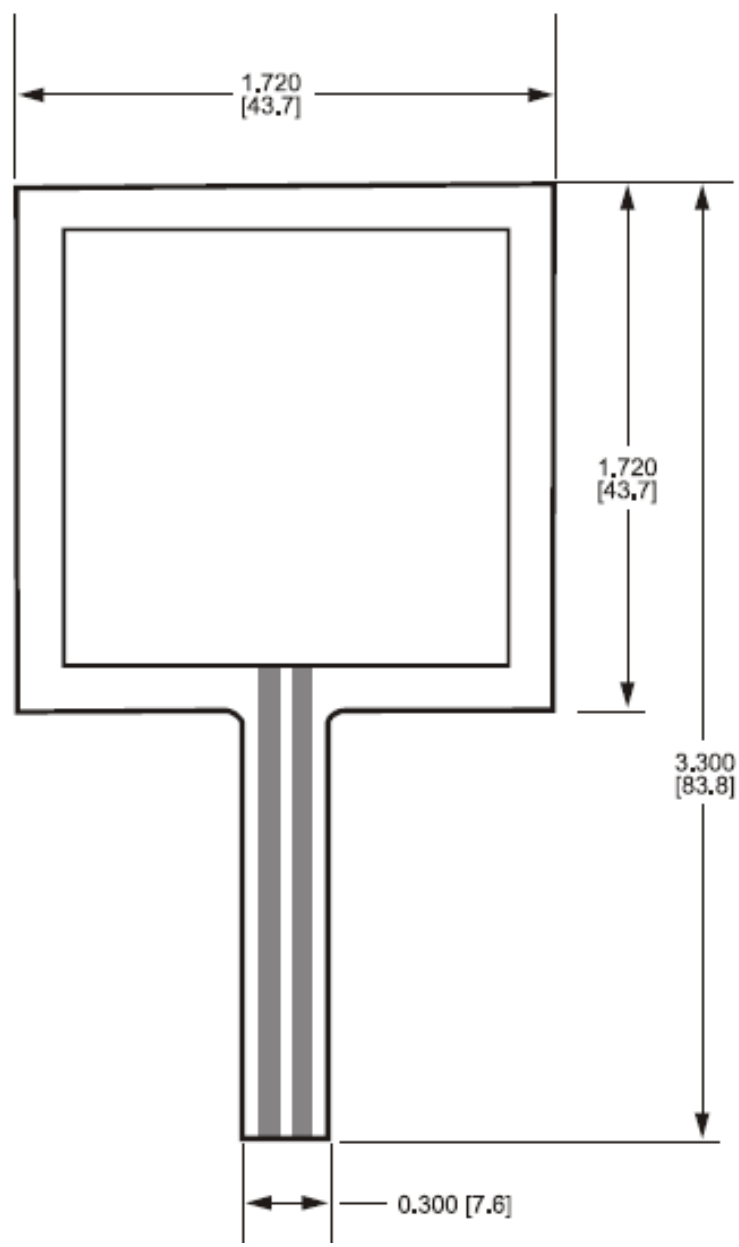

Active Area: $1.5 "[38.1] \times 1.5 "[38.1]$

Nominal thickness: $0.018 "$ [0.46 mm]

Material Build:

Semiconductive Layer

0.005 " [0.13] Ultem

Spacer Adhesive

0.006 " [0.15] Acrylic

Conductive Layer

0.005 " [0.13] Ultem

Rear Adhesive

0.002 " [0.05] Acrylic

Connectors

a. No connector

b. Solder Tabs (not shown)

c. AMP Female connector

Figure 5 shows the force sensor with rectangular/square active area.

\section{IV.RESULTS AND FINDINGS}

Figure 6, 7, 8 and 9 shows the readings of foot bottom in $\mathrm{N} / \mathrm{m}^{2}$. A graph of $\mathrm{N} / \mathrm{m}^{2} \mathrm{v} / \mathrm{s}$ time is plotted for different pressure points of the foot bottom. 


\section{IJARCCE}

International Journal of Advanced Research in Computer and Communication Engineering ISO 3297:2007 Certified

Vol. 6, Issue 6, June 2017

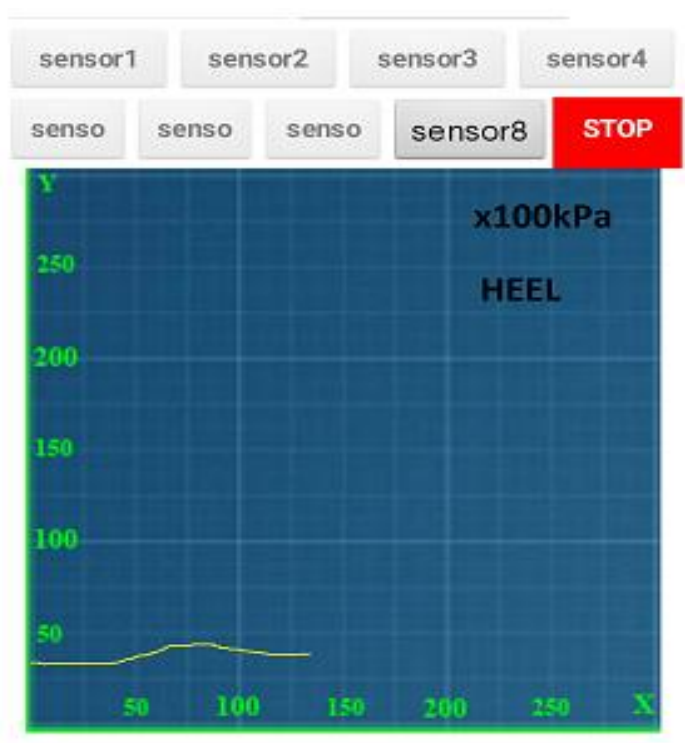

Figure 6 shows the pressure at the 'heel'.

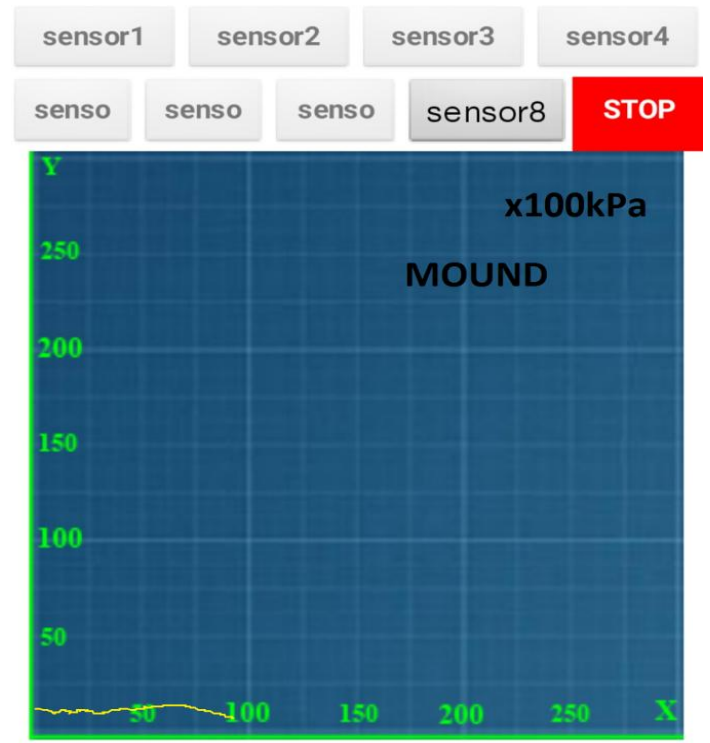

Figure 7 shows the pressure at the 'mounds'.

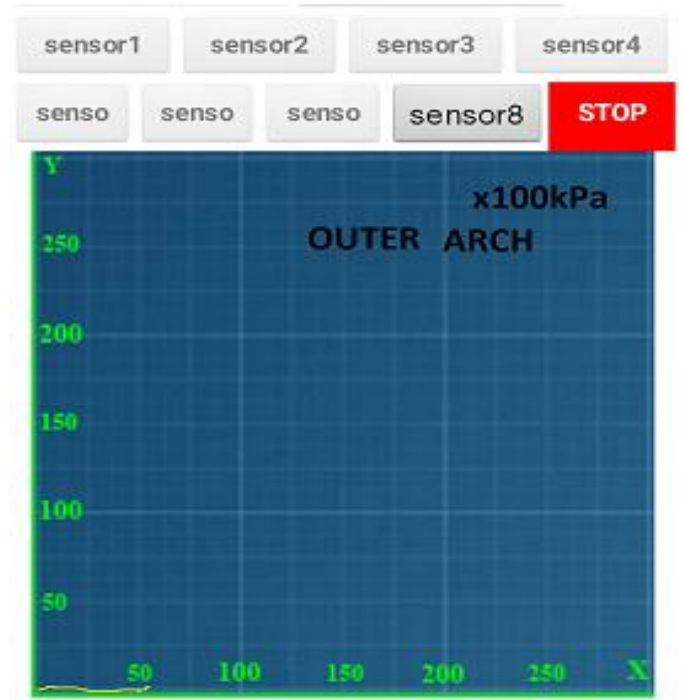

Figure 8 shows the pressure at the 'outer arch'. 
Vol. 6, Issue 6, June 2017

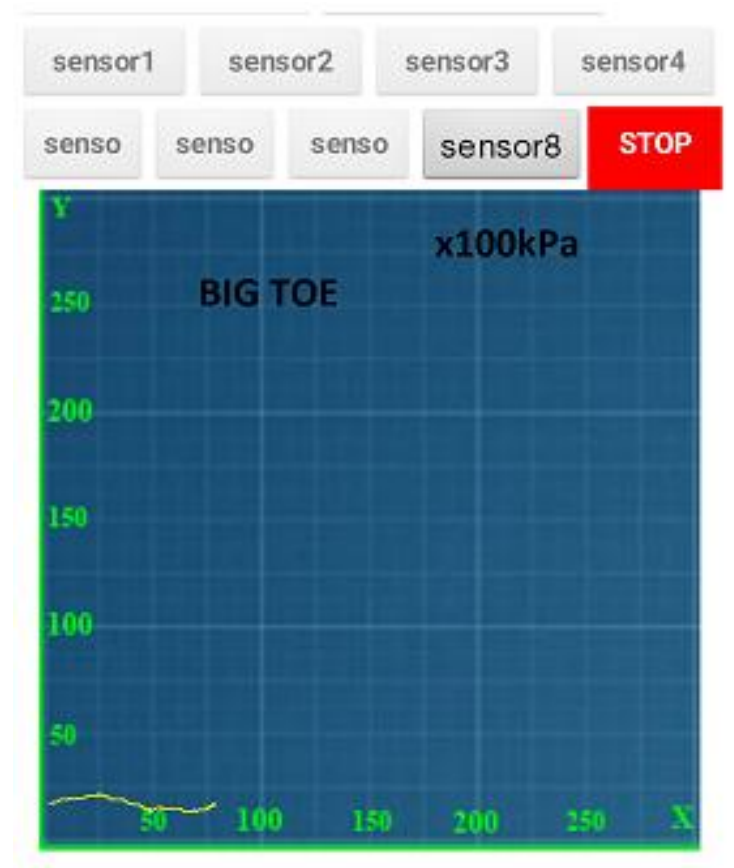

Figure 9 shows the pressure at the 'big toe'.

\section{CONCLUSIONS}

In this paper Real-time readings from the sensor array was obtained and displayed on the smartphone using the Android Application developed by us. Force/unit area of the foot was measured at various regions- toe, heel, inner and outer arch and foot mound regions. A graph of $\mathrm{N} / \mathrm{m}^{2} \mathrm{v} / \mathrm{s}$ time was plotted and recorded to the phone SD card. Each pressure point on the foot shows different values because of variations in the force applied per unit area of the foot. This analysis of differential pressure across the surface of the foot is helpful in the analysis of foot pressure in shoe manufacturing industry, performance analysis of athletes, fitness test of athletes and foot pressure analysis of diabetics to prevent ulcers.

\section{REFERENCES}

[1] Theoretical and Linearity Analysis for Pressure Sensors and ...journals.sagepub.com/doi/full/10.1155/2014/902976

[2] Sensor Terminology - National Instruments- www.ni.com > White Papers

[3] Uniformity, Repeatability, Stability, and Accuracy - Apogee Instruments-https://www.apogeeinstruments.com/uniformity-repeatabilitystability-and-accuracy/

[4] Sensor Resolution - Oregon State Geology geo.oregonstate.edu/classes/geo444_544/LECTURES/lecture5.pdf

[5] Sensors Characteristics - Springer www.springer.com/cda/content/document/cda.../9781461450511-c1.pdf?SGWID

[6] FSR Integration Guide and Evaluation Parts Catalogue with Suggested Electrical Interfaces. 